\title{
Studies on new substituted pyridazinones: synthesis and biological evaluation
}

\author{
Mohammad Asif', Anita Singh², Shah Alam Khan³, Asif Husain ${ }^{4 *}$ \\ ${ }^{1}$ Department of Pharmacy, GRD (PG) Institute of Management and Technology, Dehradun, India, ${ }^{2}$ Department of \\ Pharmaceutical Sciences, Kumaun University Campus, Bhimtal, Nainital, (UK) India, ${ }^{3}$ Department of Pharmacy, Oman \\ Medical College, Muscat, Sultanate of Oman, ${ }^{4}$ Department of Pharmaceutical Chemistry, School of Pharmaceutical Education \\ and Research, Jamia Hamdard (Hamdard University), New Delhi, India
}

\begin{abstract}
We report herein the synthesis and pharmacological evaluation of a new series of 6-aryl-2-(imidazol1-yl/1,2,4-triazol-1-yl)-2-methyl-4,5-dihydro-(2H)-pyridazin-3-one (3a-j) as potential anticonvulsant and antitubercular agents. The title compounds were prepared by reacting 6-aryl-4,5-dihydro- $(2 H)$ pyridazin-3-one (2a-e) with formaldehyde and secondary cyclic amine imidazole or 1,2,4-triazole as per Mannich reaction. Anticonvulsant activity of pyridazinone derivatives was tested at $50 \mathrm{mg} \cdot \mathrm{kg}^{-1} \mathrm{dose}$ level against maximal electroshock (MES), isoniazid (INH, $250 \mathrm{mg} \cdot \mathrm{kg}^{-1}$ ) and pentylenetetrazole (PTZ at $\left.80 \mathrm{mg} \cdot \mathrm{kg}^{-1}\right)$ induced seizure methods. Phenytoin sodium $\left(25 \mathrm{mg} \cdot \mathrm{kg}^{-1}\right)$ and sodium valproate $(100 \mathrm{mg}$. $\mathrm{kg}^{-1}$ ) were used as reference drugs for comparison purpose. In-vitro antitubercular activity was tested by Microplate Alamar Blue assay (MABA) method and the results were compared with clinically used antitubercular agents such as INH, Pyrazinamide (PZA) and Streptomycin (STM). None of the screened compounds were found to be neurotoxic at a dose level of $100 \mathrm{mg} \cdot \mathrm{kg}^{-1}$. All the screened compounds (3a-j) significantly reduced the MES, INH and PTZ induced convulsions and thus showed good anticonvulsant activity. The minimum inhibitory concentration (MIC) of the title compounds against $M$. tuberculosis ranged from $1.6 \mu \mathrm{g} / \mathrm{mL}$ to $6.25 \mu \mathrm{g} / \mathrm{mL}$ in comparison to INH, PZA $(3.125 \mu \mathrm{g} / \mathrm{mL})$ and STM $(6.25 \mu \mathrm{g} /$ $\mathrm{mL}$ ) which indicated good antitubercular activity.
\end{abstract}

Keywords: Pyridazinone/synthesis/pharmacologial evaluation. Anticonvulsant. Antitubercular. MIC.

\section{INTRODUCTION}

Epilepsy is the most common neurological disorder of central nervous system (CNS) characterized by the sudden recurrent seizures (Ahsan et al., 2012). The seizures, fits or convulsions are caused by sudden increase in the electrical activity in the brain. Epilepsy is a destructive condition that is quite prevalent in all age groups and affects millions of people worldwide. Approximately, $1-2 \%$ of total world's population is currently suffering from epilepsy and almost two million new cases of epilepsy are diagnosed every year. There are over twenty drugs available in the market for the treatment of epilepsy but only six or seven out of them are

\footnotetext{
*Correspondence: A. Husain. Department of Pharmaceutical Chemistry, School of Pharmaceutical Education and Research, Jamia Hamdard, New Delhi-110 062, India. Phone: +91-11-26059681, 26059688, ext. 5647, Mobile: +91-989-1116086. Fax: +91-11-16988874. E-mail: drasifhusain@yahoo.com; ahusain@jamiahamdard.ac.in
}

preferred in clinical setting. It has been reported that the convulsions of approximately $25 \%$ of epileptic patients are inadequately controlled by the current drug therapy (Deng et al., 2010; Edafiogho et al., 2009). Despite the development of several new anticonvulsant drugs, the treatment of epilepsy still remains inadequate.

Antiepileptic drugs (AED) should be prescribed and used carefully as these drugs can cause severe side effects eventually affecting quality of life. Their use is associated with significant dose-related toxicity and adverse effects such as tremor, blurred vision, neurotoxicity and sometimes death due to either hepatic damage or from aplastic anaemia (Ghogare et al., 2010; Gunia et al., 2010; Hu et al., 2010).

An anticonvulsant drug acts by multiple mechanism involving interaction with more than one specific target. Therefore, evaluation of anticonvulsant drugs mostly includes in vivo screening tests employing different seizure models which have been developed to investigate 
the diverse mechanisms of action of potential drug molecules. There is diversity in the structures of currently available drugs for the management of epilepsy like phenytoin, lamotrigine, phenobarbitone, rufinamide and carbamazepine. The anticonvulsant properties of these drugs could possibly be attributed to the presence of diverse group of chemical functionalities like hydrazones $(=\mathrm{N}-\mathrm{NH}-)$, amides $\left(-\mathrm{CONH}_{2}\right)$, and carbamides (-NHCONH-) etc., present in these molecules.

Tuberculosis (TB), caused by Mycobacterium tuberculosis, is a highly infectious disease affecting approximately one third of the world's population. It kills almost 2 million people each year across the globe. Despite the availability of antitubercular drugs since $1950 \mathrm{~s}, \mathrm{~TB}$ continues to be one of the top three infectious diseases of global concern (Sandhu, 2011). The current situation is worsened by HIV epidemic that has resulted in high incidence rates of developing active $\mathrm{TB}$, an increased prevalence of multi-drug resistant TB (MDR-TB) and an ever increasing emergence of extensively drug-resistant (XDR) TB (Asif, 2012a). The development of resistance makes TB difficult to cure with the available drugs which particularly interfere and inhibit cell processes of growing bacteria. Therefore, there is an urgent need to develop new TB drugs having both bactericidal as well as sterilizing activity against TB bacillus (Asif, 2012b). Sincere efforts should be made to discover and develop anti TB drugs that are: able to shorten the duration of therapy; active against resistant TB i.e. MDR and XDR strains; free from hepatotoxicity; having easy to metabolize chemical structures and which can be taken along with HIV medications, etc.

The pyridazinones belong to an important class of heterocyclic compounds which have been reported to exhibit several useful biological activities such as antibacterial, antifungal, antidepressant, antihypertensive, anti platelets, cardio tonic, diuretics, anti-HIV, anticancer, antiulcer and other useful anticipated activities (Akahane, Katayama, Mitsunaga, 1999; Amin et al., 2010; Asif, 2012a; Demirayak, Karaburun, Beis, 2004; Khokra et al., 2015). Some pyridazinone compounds are used as drugs like indolidan, pimobendan, bemoradan, primobendan, levosimendan and azanrinone (cardiovascular agents), minaprine (antidepressant), emorfazone (antiinflammatory) (Asif, 2012b). Literature revealed that various pyridazinone analogues are active against epilepsy (Hallot et al., 1986; Siddiqui et al., 2006; Xu et al., 1991) and Mtb (Islam, Siddiqui, Rajesh, 2008; Siddiqui, Mishra, Shaharyar, 2010).

Isoniazid (INH) generated convulsions and anti-TB effects could be related to its structure which contains an aromatic pyridinyl ring and a free carbonyl hydrazide group at para position (Figure 1). Similarly, it has been reported that aryl pyridazinone compounds bearing an aromatic group at 6-position and a cyclic carbonyl hydrazide group as a part of its ring system exhibits anticonvulsant and antitubercular activities (Asif, Singh, Bilkanti, 2013). Thus, on the basis of structural similarities between INH and aryl pyridazinone, we deduced that cyclic carbonyl hydrazide group is necessary for anticonvulsant activity (ACA) in pyridazine ring while open carbonyl hydrazide group is essential for anti-TB activity. These structural similarities encouraged us to prepare new compounds having both functionalities in search of compounds exhibiting both antiepileptic and antitubercular properties (Bialer, 2006; Gerlach, Krajewski, 2010; Asif, Singh, 2015).

The aryl-pyridazinone moiety is made up of several smaller functional groups exhibiting anti-TB activity (Figure 2). These aryl-pyridazinones contain carbonyl hydrazide group in cyclic structure and aryl group, similar groups are also present in well known anti-TB drug, isoniazid. Considering the 6-aryl-pyridazinone moiety as the pharmacophoric group for the biological activity, we have synthesized some 6-aryl-2-(imidzol-1yl or (1,2,4-triazol-1-yl)-2-methyl-pyridazin-3(2H)-one derivatives and evaluated them for anticonvulsant activity by maximum electro shock (MES), isoniazid (INH)
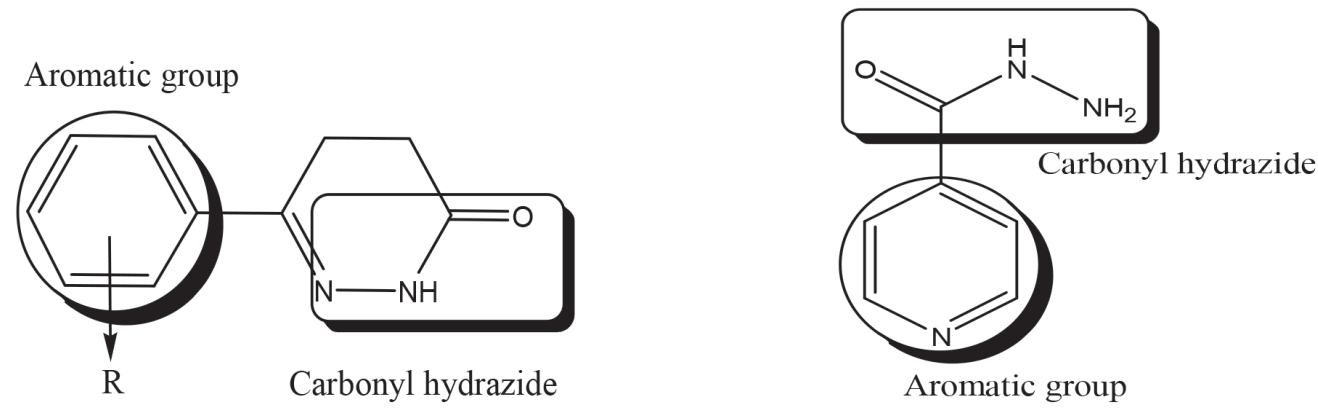

FIGURE 1 - Structure similarities of Aryl-pyridazinone compounds and anti-TB drug Isoniazid. 
and subcutaneous pentylenetetrazole (scPTZ) induced convulsion methods and in-vitro antitubercular activity by microplate alamar blue dye assay (MABA) method.

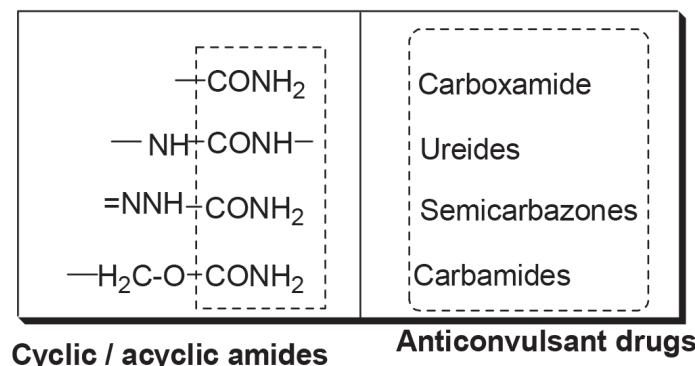

\begin{tabular}{|c|c|}
\hline $\begin{array}{r:r}\text { Pyridyl } & \mathrm{CO}-\mathrm{NH}-\mathrm{NH}_{2} \\
\text { Piperazinyl } & \mathrm{CO}-\mathrm{NH}_{2}\end{array}$ & $\begin{array}{l}\text { Isoniazid \& Aconiazid } \\
\text { Pyrizinamide \& } \\
\text { Streptomycine }\end{array}$ \\
\hline
\end{tabular}

FIGURE 2 - Concept of pyridazinones as anticonvulsants and as anti-TB agents.

\section{MATERIAL AND METHODS}

\section{Chemistry}

All the chemicals used were of high purity and were purchased from Central Drug House $(\mathrm{CDH})$, Loba Chem and Hi-media. 6-(aryl)-2-(imidazol-1-yl/1,2,4triazol-1-yl-methyl)-4,5- dihydro $(2 H)$-pyridazin-3-one derivatives were prepared according to the Scheme 1. The Friedel-Craft acylation of substituted aromatic compound with succinic anhydride in presence of anhydrous aluminum chloride $\left(\mathrm{AlCl}_{3}\right)$ yielded the substituted 4-oxo-butyric acid (1a-e). The resulting aroyl propionic acids on reaction with hydrazine hydrate gave the 6-aroyl-pyridazinones (2a-e). 6-aryl-pyridazinones $(2 \mathrm{mM})$ were mixed with cyclic secondary amine (imidazole and 1,2,4-triazole; $2 \mathrm{mM}$ ), formaldehyde $(40 \mathrm{mM})$ and refluxed on a water bath for 8-12 h to obtain the final compounds (3a-j) by Mannich reaction. An open tube capillary method was used to determine melting point of synthesized compounds and are uncorrected. Purity of the compounds was checked by silica gel G coated thin layer chromatography (TLC) plates. The spots on TLC plates were detected under UV light and by exposing to iodine vapors. The FT-IR spectra were recorded on Bio-rad FTS-135 spectrophotometer using $\mathrm{KBr}$ pellets; ${ }^{1} \mathrm{H}$ NMR and ${ }^{13} \mathrm{CNMR}$ spectra were recorded on Bruker Spectrospin DPX $300 \mathrm{MHz}$ using DMSO- $\mathrm{d}_{6}$ and $\mathrm{CDCl}_{3}$ as solvent. Chemical shift values are reported as $\delta(\mathrm{ppm})$ and coupling constants ( $J$ values) are expressed in $\mathrm{Hz}$ with reference to tetramethyl silane (TMS). Mass spectrometry for title compounds was performed on a JEOL JMS-D 300 instrument fitted with a JMS 2000 data system at $70 \mathrm{eV}$.

\section{Synthesis of aroyl propionic acid (1a-e)}

All the aroyl propionic acids (1a-e) were synthesized as per the previously reported procedure with slight adjustment in temperature of reaction, duration of reaction and by using nitrobenzene as a solvent (Siddiqui, Mishra, Shaharyar, 2010). Briefly, an equimolar quantity of aromatic compound (toluene, naphthalene, ethylbenzene, xylene, biphenyl; $0.1 \mathrm{~mol}$ ) and succinic anhydride $(0.1 \mathrm{~mol})$ were dissolved in nitrobenzene and were refluxed in presence of anhydrous aluminium chloride $(0.1125 \mathrm{~mol})$ on a water bath for two- four hours. The excess solvent was removed by steam distillation. It was

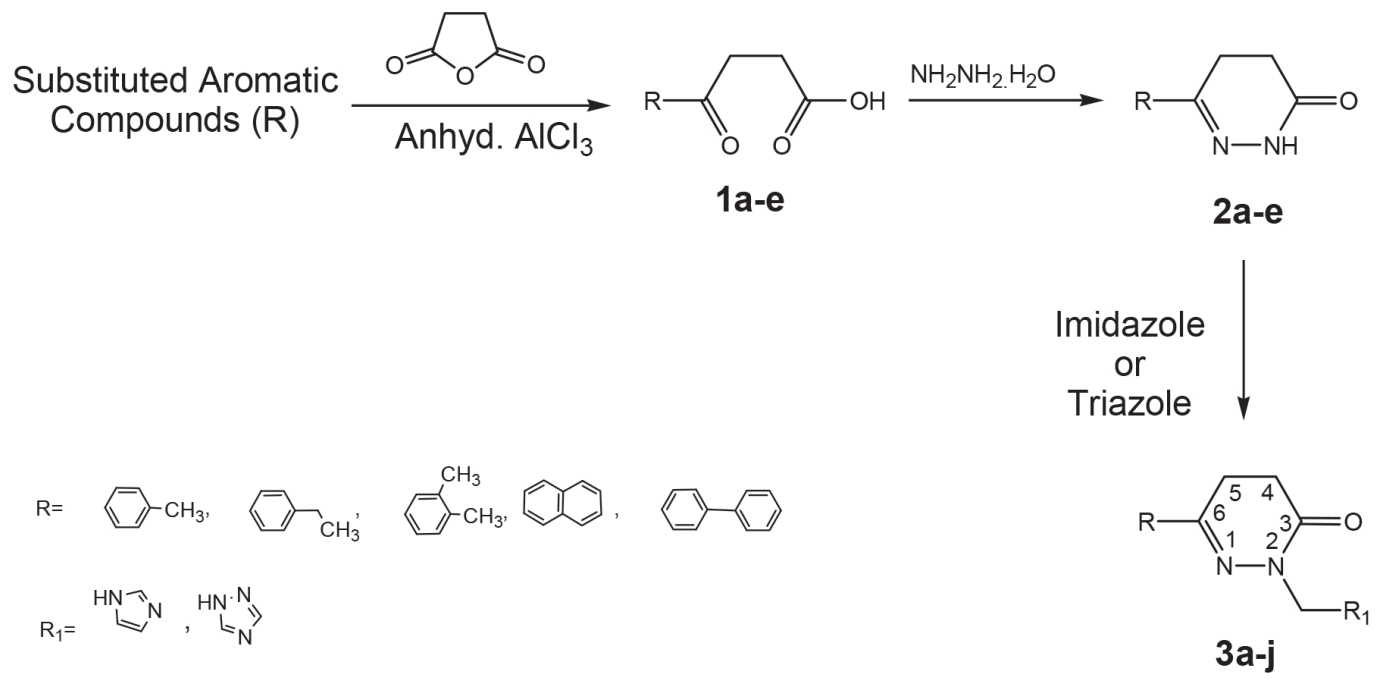

SCHEME 1- Synthetic route for the synthesis of target compounds (3a-j) 
purified by dissolving in sodium hydroxide solution, filtering, followed by the addition of hydrochloric acid. The solid mass so obtained was filtered, washed with cold water, dried and re-crystallized from ethanol to obtain aroyl propionic acid.

\section{4-p-tolyl-4-oxobutyric acid (1a)}

mp $122-123^{\circ} \mathrm{C} ; 67 \%$ yield. IR: $\left(\mathrm{KBr}, \mathrm{cm}^{-1}\right): 1659$ (CO), 2996 (CH), 3413 (OH); ${ }^{1} \mathrm{H}-\mathrm{NMR}$ (DMSO-d 6 , $\delta$ ppm): $2.38\left(\mathrm{~s}, 3 \mathrm{H}, \mathrm{CH}_{3}\right), 2.78\left(\mathrm{t}, 2 \mathrm{H}, \mathrm{CH}_{2}\right), 2.99(\mathrm{t}, 2 \mathrm{H}$, $\left.\mathrm{CH}_{2}\right), 7.26$ (t, 2H, Ar-H3',5'), 12.15 (s, 1H, COOH); MS $(\mathrm{m} / \mathrm{z}): 193\left(\mathrm{M}^{+}+1 ; \mathrm{C}_{11} \mathrm{H}_{12} \mathrm{O}_{3}\right)$.

\section{4-naphthalen-2-yl-4-oxobutyric acid (1b)}

mp $116-117^{\circ} \mathrm{C} ; 62 \%$ yield. IR: $\left(\mathrm{KBr}, \mathrm{cm}^{-1}\right): 1705$ (CO), 3096 (CH), 3401. (OH); ${ }^{1} \mathrm{H}-\mathrm{NMR}$ (DMSO-d ${ }_{6}, \delta$ ppm): 2.80 (t, 2H, $\left.\mathrm{CH}_{3}\right), 3.11\left(\mathrm{t}, 2 \mathrm{H}, \mathrm{CH}_{2}\right), 7.26-8.3$ (m, $7 \mathrm{H}, \mathrm{Ar}-\mathrm{H}), 12.15(\mathrm{~s}, 1 \mathrm{H}, \mathrm{COOH}) ; \mathrm{MS}(\mathrm{m} / \mathrm{z}): 228\left(\mathrm{M}^{+}\right.$; $\mathrm{C}_{14} \mathrm{H}_{12} \mathrm{O}_{3}$ ).

\section{4-p-ethylphenyl-4-oxobutyric acid (1c)}

mp $138-140{ }^{\circ} \mathrm{C} ; 73 \%$ yield. IR: $\left(\mathrm{KBr}, \mathrm{cm}^{-1}\right): 1698$ (CO), $2990.6(\mathrm{CH}), 3402.6(\mathrm{OH})$; ${ }^{1} \mathrm{H}-\mathrm{NMR}$ (DMSO-d ${ }_{6}$, $\delta \mathrm{ppm}): 1.24\left(\mathrm{t}, 3 \mathrm{H}, \mathrm{CH}_{3}\right), 2.79\left(\mathrm{q}, 2 \mathrm{H}, \mathrm{CH}_{2}\right), 2.40(\mathrm{t}, 2 \mathrm{H}$, $\mathrm{CH}_{3}$ ), 3.20 (t, 2H, $\mathrm{CH}_{2}$ ), 7.24 (m, 2H, Ar-H3', H5'), 7.84 (m, 2H, Ar-H2', H6'), 12.15 (s, 1H, COOH); MS (m/z): $207\left(\mathrm{M}^{+}+1 ; \mathrm{C}_{12} \mathrm{H}_{14} \mathrm{O}_{3}\right)$.

\section{4(3,4-dimethylphenyl)- 4-oxobutyric acid (1d)}

mp $145^{\circ} \mathrm{C} ; 45 \%$ yield. IR: $\left(\mathrm{KBr}, \mathrm{cm}^{-1}\right)$ : $1718(\mathrm{CO})$, 3105.6 (CH), 3410. (OH); ${ }^{1} \mathrm{H}-\mathrm{NMR}$ (DMSO-d ${ }_{6}, \delta$ ppm): $2.35\left(\mathrm{~s}, 6 \mathrm{H}, 2 \mathrm{xCH}_{3}\right), 2.80\left(\mathrm{t}, 2 \mathrm{H}, \mathrm{CH}_{2}\right), 3.10\left(\mathrm{t}, 2 \mathrm{H}, \mathrm{CH}_{2}\right)$, 7.20-7.59 (m, 3H, Ar-H2', H3', H5'), 12.10 (s, 1H, $\mathrm{COOH}) ; \mathrm{MS}(\mathrm{m} / \mathrm{z}): 207\left(\mathrm{M}^{+}+1 ; \mathrm{C}_{12} \mathrm{H}_{14} \mathrm{O}_{3}\right)$.

\section{4-biphenyl 4-oxobutyric acid (1e)}

mp $127-128{ }^{\circ} \mathrm{C} ; 63 \%$ yield. IR: $\left(\mathrm{KBr}, \mathrm{cm}^{-1}\right): 1720$ (CO), $2905.6(\mathrm{OH}), 3410.6$ (NH); ${ }^{1} \mathrm{H}-\mathrm{NMR}$ (DMSO-d ${ }_{6}$, $\delta \mathrm{ppm}): 2.35\left(\mathrm{~s}, 3 \mathrm{Hx} 2, \mathrm{CH}_{3}\right), 2.84\left(\mathrm{t}, 2 \mathrm{H}, \mathrm{CH}_{3}\right), 3.0(\mathrm{t}, 2 \mathrm{H}$, $\left.\mathrm{CH}_{2}\right), 7.22-8.50(\mathrm{~m}, 9 \mathrm{H}, \mathrm{Ar}-\mathrm{H}), 12.10(\mathrm{~s}, 1 \mathrm{H}, \mathrm{COOH}) ; \mathrm{MS}$ $(\mathrm{m} / \mathrm{z}): 255\left(\mathrm{M}^{+}+1 ; \mathrm{C}_{16} \mathrm{H}_{14} \mathrm{O}_{3}\right)$.

Synthesis of 6-aryl 2,3,4,5-tetrahydropyridazin-3-one (2a-e)

These compounds were synthesized as per the reported method (Islam, Siddiqui, Rajesh, 2008; Siddiqui, Mishra, Shaharyar, 2010).

\section{6-(p-tolyl-4,5-dihydro(2H)- pyridazin-3-one (2a)}

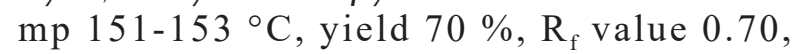
IR:(KBr, $\left.\mathrm{cm}^{-1}\right): 1659(\mathrm{C}=\mathrm{O}), 3217(\mathrm{NH}), 1511(\mathrm{C}=\mathrm{N})$;
${ }^{1} \mathrm{HNMR}\left(\mathrm{DMSO}-\mathrm{d}_{6}, \delta, \mathrm{ppm}\right): 2.36\left(\mathrm{~s}, 3 \mathrm{H}, \mathrm{CH}_{3}\right), 2.60(\mathrm{t}$, 2H, $\mathrm{CH}_{2}$ ), 2.97 (t, 2H, $\mathrm{CH}_{2}$ ), 7.27 (t, 2H, Ar-H3',5'), 7.65 (d,2H, Ar-H2',6'), 8.80 (s, H, NH); MS (m/z): $188\left(\mathrm{M}^{+}\right.$; $\mathrm{C}_{11} \mathrm{H}_{12} \mathrm{~N}_{2} \mathrm{O}$ ).

\section{6-(naphthalen-2-yl)-4,5-dihydro(2H)-pyridazin-3-one} (2b)

mp 165-166 ${ }^{\circ} \mathrm{C}$; yield $75 \%$, $\mathrm{R}_{\mathrm{f}}$ value 0.65 , IR: $(\mathrm{KBr}$, $\left.\mathrm{cm}^{-1}\right): 3094 \mathrm{~cm}^{-1}(\mathrm{CH}), 1708 \mathrm{~cm}^{-1}(\mathrm{C}=\mathrm{O}), 1617 \mathrm{~cm}^{-1}(\mathrm{C}=\mathrm{C})$. ${ }^{1} \mathrm{HNMR}\left(\mathrm{DMSO}-\mathrm{d}_{6}\right) \mathrm{ppm} 3.1\left(\mathrm{t}, 2 \mathrm{H}, \mathrm{CH}_{2}\right), 3.36(\mathrm{t}, 2 \mathrm{H}$, $\mathrm{CH}_{2}$ ), 7.29- 8.2 (m, 7H, Ar-H), 8.82 (s, H, NH); MS (m/z): $224\left(\mathrm{M}^{+} ; \mathrm{C}_{14} \mathrm{H}_{12} \mathrm{~N}_{2} \mathrm{O}\right)$

\section{6-(4-ethylphenyl)-4,5-dihydro(2H)-pyridazin-3-one} (2c)

mp 119-122 ${ }^{\circ} \mathrm{C}$; yield $70 \%, \mathrm{R}_{\mathrm{f}}$ value 0.73 , IR: $(\mathrm{KBr}$, $\left.\mathrm{cm}^{-1}\right): 1666(\mathrm{C}=\mathrm{O}), 3428(\mathrm{NH}), 1595(\mathrm{C}=\mathrm{N})$; ${ }^{1} \mathrm{HNMR}$ (DMSO-d $\left.{ }_{6}, \delta, \mathrm{ppm}\right): 1.24\left(\mathrm{t}, 3 \mathrm{H}, J=7 \mathrm{~Hz}, \mathrm{CH}_{3}-\mathrm{CH}_{2^{-}}\right)$, $1.59\left(\mathrm{q}, 2 \mathrm{H}, \mathrm{CH}_{3}-\mathrm{C}_{2}-\mathrm{s}\right), 2.64\left(\mathrm{~m}, 2 \mathrm{H}, \mathrm{CH}_{2}\right), 3.01(\mathrm{~m}, 2 \mathrm{H}$, $\mathrm{CH}_{2}$ ), 7.26 (d, 2H, Ar-H3',5') 7.65 (d, 2H, Ar-H2',6'), 8.54 (s, H, NH); MS (m/z): $202\left(\mathrm{M}^{+} ; \mathrm{C}_{10} \mathrm{H}_{11} \mathrm{NO}_{3}\right)$.

\section{6-(3,4-dimethylphenyl)-4,5- dihydro(2H)-pyridazin-3-} one $(\mathbf{2 d})$

mp $162-163{ }^{\circ} \mathrm{C}$, yield $50 \%, \mathrm{R}_{\mathrm{f}}$ value 0.50 , IR: $(\mathrm{KBr}$, $\left.\mathrm{cm}^{-1}\right): 1668(\mathrm{C}=\mathrm{O}), 3220(\mathrm{NH}), 1508(\mathrm{C}=\mathrm{N})$; ${ }^{1} \mathrm{HNMR}$ $\left(\mathrm{DMSO}_{6} \mathrm{~d}_{6}, \delta, \mathrm{ppm}\right): 1.36\left(\mathrm{~s}, 6 \mathrm{H}, 2 \mathrm{xCH}_{3}\right), 2.65(\mathrm{t}, 2 \mathrm{H}$, $\mathrm{CH}_{2}$ ), 3.01 (t, 2H, $\left.\mathrm{CH}_{2}\right), 7.47$ (d, H, ArH5'), 7.65 (d, H, Ar-H6'), 7.51 (s, H, Ar-H2'), 8.70 (s, H, NH); MS (m/z): $202\left(\mathrm{M}^{+} ; \mathrm{C}_{12} \mathrm{H}_{14} \mathrm{~N}_{2} \mathrm{O}\right)$.

6-(1,1'-biphenyl-4-yl)-4,5-dihydro(2H) pyridazin-3-one (2e)

mp 240-243 ${ }^{\circ} \mathrm{C}$, yield $90 \%, \mathrm{R}_{\mathrm{f}}$ value 0.72 , IR: $(\mathrm{KBr}$, $\left.\mathrm{cm}^{-1}\right): 3248(\mathrm{NH}), 1700(\mathrm{C}=\mathrm{O}) ;{ }^{1} \mathrm{HNMR}\left(\mathrm{DMSO}-\mathrm{d}_{6}\right.$, $\delta, \mathrm{ppm}): 2.7\left(\mathrm{t}, 2 \mathrm{H}, \mathrm{CH}_{2}\right), 2.98\left(\mathrm{t}, 2 \mathrm{H}, \mathrm{CH}_{2}\right), 7.39-7.83$ (m, 9H, Ar-H), 9.94 (s, 1H, NH); MS (m/z): $250\left(\mathrm{M}^{+}\right.$; $\mathrm{C}_{16} \mathrm{H}_{14} \mathrm{~N}_{2} \mathrm{O}$ ).

General procedure for the synthesis of 6-aryl-2(imidzol-1yl / 1,2,4-triazol-1-yl)-2-methyl-4,5dihydro(2H)-pyridazin-3-one (3a-j)

A mixture of 6-ary14,5-dihydropyridazin-3-one (2a-e; $0.002 \mathrm{~mol})$, formaldehyde $37-41 \%(0.04 \mathrm{~mol})$ and an appropriate secondary amine $(0.002 \mathrm{~mol})$ in ethanol $(50 \mathrm{~mL})$ was allowed to stand overnight at the room temperature and then refluxed for 8-12 h. The progress of reaction was monitored by TLC. The product which was left after removal of most of the solvent was poured into crushed ice. The solid so obtained was crystallized from ethanol to afford pure solid product. 
2-(1,2,4)-triazol-1-yl-methyl-6-(4-ethylphenyl)-4,5-

dihydro (2H)-pyridazin-3-one (3a)

Yield: $50 \%$; mp $140-142{ }^{\circ} \mathrm{C}$; IR $\left(\mathrm{KBr}, \mathrm{cm}^{-1}\right): 2995$ $(\mathrm{CH}), 1670(\mathrm{C}=\mathrm{O}) .{ }^{1} \mathrm{H}$ NMR $\left(\mathrm{CDCl}_{3}\right) \delta(\mathrm{ppm}): 1.2(\mathrm{t}, 3 \mathrm{H}$, $\left.\mathrm{CH}_{3}\right), 2.5\left(\mathrm{q}, 2 \mathrm{H}, \mathrm{CH}_{2}\right), 2.6\left(\mathrm{t}, 2 \mathrm{H}, \mathrm{C}-\mathrm{CH}_{2}\right), 3.0(\mathrm{t}, 2 \mathrm{H}$, $\left.-\mathrm{CH}_{2}-\mathrm{CO}\right), 5.3\left(\mathrm{~s}, 2 \mathrm{H},-\mathrm{N}-\mathrm{CH}_{2}-\mathrm{N}-\right), 7.3-7.9(\mathrm{~m}, 6 \mathrm{H}$, $\mathrm{Ar}-\mathrm{H}) ;{ }^{13} \mathrm{C} \mathrm{NMR}\left(\mathrm{CDCl}_{3}\right) \delta(\mathrm{ppm}): 15.5\left(\mathrm{CH}_{3}\right), 25.5-65.2$ $\left(4 \mathrm{XCH}_{2}\right), 125.1-152.8(\mathrm{Ar}-\mathrm{C}), 173.5(\mathrm{C}=\mathrm{O}) ; \mathrm{MS}(\mathrm{m} / \mathrm{z})$ : $283\left(\mathrm{M}^{+} ; \mathrm{C}_{15} \mathrm{H}_{17} \mathrm{~N}_{5} \mathrm{O}\right)$.

6-biphenyl-4-yl-2-imidazol-1-yl-methyl-4,5-

dihydro $(2 \mathrm{H})$-pyridazin-3-one (3b)

Yield: $50 \%$; mp $140-142{ }^{\circ} \mathrm{C}$; IR $\left(\mathrm{KBr}, \mathrm{cm}^{-1}\right): 2990$ $(\mathrm{CH}), 1678(\mathrm{C}=\mathrm{O}) .{ }^{1} \mathrm{H}$ NMR $\left(\mathrm{CDCl}_{3}\right) \delta(\mathrm{ppm}): 2.6(\mathrm{t}, 2 \mathrm{H}$, $\left.\mathrm{C}-\mathrm{CH}_{2}\right), 3.11\left(\mathrm{t}, 2 \mathrm{H}, \mathrm{CH}_{2}-\mathrm{CO}\right), 5.27\left(\mathrm{~s}, 2 \mathrm{H},-\mathrm{N}-\mathrm{CH}_{2}-\mathrm{N}-\right)$, 7.36-8.14 (m, $11 \mathrm{H}, \mathrm{Ar}-\mathrm{H}) ;{ }^{13} \mathrm{C}$ NMR $\left(\mathrm{CDCl}_{3}\right) \delta(\mathrm{ppm})$ : 28.4, 33.4, 67.2 $\left(3 \mathrm{XCH}_{2}\right), 125.9-157.1$ (Ar-C), 176.1 $(\mathrm{C}=\mathrm{O}) ; \mathrm{MS}(\mathrm{m} / \mathrm{z}): 330\left(\mathrm{M}^{+} ; \mathrm{C}_{20} \mathrm{H}_{18} \mathrm{~N}_{4} \mathrm{O}\right)$.

6-biphenyl-4-yl-2(1,2,4)triazol-1-yl-methyl-4,5dihydro $(2 \mathrm{H})$-pyridazin-3-one (3c)

Yield: $61 \%$; mp $145-146^{\circ} \mathrm{C}$; IR $\left(\mathrm{KBr}, \mathrm{cm}^{-1}\right): 2992$ $(\mathrm{CH}), 1690(\mathrm{C}=\mathrm{O}), 1590(\mathrm{C}=\mathrm{N}) .{ }^{1} \mathrm{H}$ NMR $\left(\mathrm{CDCl}_{3}\right) \delta$ (ppm): 2.6 (t, 2H, C- $\mathrm{CH}_{2}$ ), 2.96 (t, 2H, $\left.\mathrm{CH}_{2}-\mathrm{CO}\right), 5.26$ (s, 2H, $\left.\left.-\mathrm{N}-\mathrm{CH}_{2}-\mathrm{N}-\right), 7.40-7.81(\mathrm{~m}, 12 \mathrm{H}, \mathrm{Ar}-\mathrm{H}) ;\right) ;{ }^{13} \mathrm{C}$ $\mathrm{NMR}\left(\mathrm{CDCl}_{3}\right) \delta(\mathrm{ppm}): 26.1-65.2\left(3 \mathrm{XCH}_{2}\right), 127.2-152.5$ $(\operatorname{Ar}-\mathrm{C}), 173(\mathrm{C}=\mathrm{O}) ; \mathrm{MS}(\mathrm{m} / \mathrm{z}): 331\left(\mathrm{M}^{+} ; \mathrm{C}_{19} \mathrm{H}_{17} \mathrm{~N}_{5} \mathrm{O}\right)$.

6-(4-ethyl-phenyl)-2-imidazol-1-yl-methyl-4,5dihydro(2H)-pyridazin-3-one (3d)

Yield: $53 \%$; mp $135-137^{\circ} \mathrm{C}$; IR $\left(\mathrm{KBr}, \mathrm{cm}^{-1}\right): 3005$ $(\mathrm{CH}), 1685(\mathrm{C}=\mathrm{O}) .{ }^{1} \mathrm{H}$ NMR $\left(\mathrm{CDCl}_{3}\right) \delta(\mathrm{ppm}): 1.2(\mathrm{t}, 3 \mathrm{H}$, $\left.\mathrm{CH}_{3}\right), 2.5\left(\mathrm{q}, 2 \mathrm{H}, \mathrm{CH}_{2}\right), 2.68\left(\mathrm{t}, 2 \mathrm{H}, \mathrm{C}-\mathrm{CH}_{2}\right), 3.0(\mathrm{t}, 2 \mathrm{H}$, $\left.\mathrm{CH}_{2}-\mathrm{CO}\right), 5.3\left(\mathrm{~s}, 2 \mathrm{H},-\mathrm{N}-\mathrm{CH}_{2}-\mathrm{N}-\right), 7.38-7.73(\mathrm{~m}, 7 \mathrm{H}$, $\mathrm{Ar}-\mathrm{H}) ;{ }^{13} \mathrm{C} \mathrm{NMR}\left(\mathrm{CDCl}_{3}\right) \delta(\mathrm{ppm}): 18.2\left(\mathrm{CH}_{3}\right), 27.4,29.5$, 33.2, $65.6\left(4 \mathrm{XCH}_{2}\right), 128.1-156.1(\mathrm{Ar}-\mathrm{C}), 175.8(\mathrm{C}=\mathrm{O})$; MS (m/z): $282\left(\mathrm{M}^{+} ; \mathrm{C}_{16} \mathrm{H}_{18} \mathrm{~N}_{4} \mathrm{O}\right)$.

\section{2-imidazol-1-yl-methyl-6-naphthalen-2-yl-4,5-} dihydro $(2 \mathrm{H})$-pyridazin-3-one (3e)

Yield: $47 \%$; mp $145-147^{\circ} \mathrm{C}$; IR $\left(\mathrm{KBr}, \mathrm{cm}^{-1}\right): 3015$ $(\mathrm{CH}), 1694(\mathrm{C}=\mathrm{O}) .{ }^{1} \mathrm{H} \mathrm{NMR}\left(\mathrm{CDCl}_{3}\right) \delta(\mathrm{ppm}): 2.64(\mathrm{t}, 2 \mathrm{H}$, $\left.\mathrm{C}-\mathrm{CH}_{2}\right), 2.95\left(\mathrm{t}, 2 \mathrm{H}, \mathrm{CH}_{2}-\mathrm{CO}\right), 5.28\left(\mathrm{~s}, 2 \mathrm{H},-\mathrm{N}-\mathrm{CH}_{2}-\mathrm{N}-\right)$, 6.8-8.3 (m, $10 \mathrm{H}, \mathrm{Ar}-\mathrm{H}) ;{ }^{13} \mathrm{C} \mathrm{NMR}\left(\mathrm{CDCl}_{3}\right) \delta(\mathrm{ppm}): 27.6$, 35.1, 65.2 $\left(3 \mathrm{XCH}_{2}\right), 123.1-155.2(\mathrm{Ar}-\mathrm{C}), 175.2(\mathrm{C}=\mathrm{O})$; $\operatorname{MS}(\mathrm{m} / \mathrm{z}): 304\left(\mathrm{M}^{+} ; \mathrm{C}_{18} \mathrm{H}_{16} \mathrm{~N}_{4} \mathrm{O}\right)$.

6-(3,4-dimethyl-phenyl)-2-imidazol-1-yl-methyl-4,5dihydro(2H)-pyridazin-3-one ( $3 \mathbf{f})$

Yield: $40 \%$; mp $131-133{ }^{\circ} \mathrm{C}$; IR $\left(\mathrm{KBr}, \mathrm{cm}^{-1}\right): 2990$ $(\mathrm{CH}), 1690(\mathrm{C}=\mathrm{O}) .{ }^{1} \mathrm{H}$ NMR $\left(\mathrm{CDCl}_{3}\right) \delta(\mathrm{ppm}): 2.29(\mathrm{~s}$, $\left.6 \mathrm{H}, 2 \mathrm{xCH}_{3}\right), 2.6\left(\mathrm{t}, J=7.5,2 \mathrm{H}, \mathrm{C}-\mathrm{CH}_{2}\right), 3.0(\mathrm{t}, J=7.5$, $\left.2 \mathrm{H}, \mathrm{CH}_{2}-\mathrm{CO}\right), 5.3\left(\mathrm{~s}, 2 \mathrm{H},-\mathrm{N}-\mathrm{CH}_{2}-\mathrm{N}-\right), 6.8-7.5(\mathrm{~m}, 6 \mathrm{H}$, $\mathrm{Ar}-\mathrm{H}) ;{ }^{13} \mathrm{C} \mathrm{NMR}\left(\mathrm{CDCl}_{3}\right) \delta(\mathrm{ppm}): 15.5\left(2 \mathrm{XCH}_{3}\right), 27.4$, 33.4, $65.1\left(3 \mathrm{XCH}_{2}\right), 125.1-155.9(\mathrm{Ar}-\mathrm{C}), 175.2(\mathrm{C}=\mathrm{O})$; $\operatorname{MS}(\mathrm{m} / \mathrm{z}): 282\left(\mathrm{M}^{+} ; \mathrm{C}_{16} \mathrm{H}_{18} \mathrm{~N}_{4} \mathrm{O}\right)$.

\section{2-(1,2,4-triazol-1-yl-methyl)-6-(3,4-}

dimethylphenyl)-4,5- dihydro(2H)-pyridazin-3-one (3g) Yield: $55 \%$; mp $126-128^{\circ} \mathrm{C}$; IR $\left(\mathrm{KBr}, \mathrm{cm}^{-1}\right): 3005$ $(\mathrm{CH}), 1685(\mathrm{C}=\mathrm{O}), 1585(\mathrm{C}=\mathrm{N}) .{ }^{1} \mathrm{H}$ NMR $\left(\mathrm{CDCl}_{3}\right) \delta$ (ppm): 2.32 (s, 6H, 2xCH $\mathrm{xCH}_{3}, 2.66\left(\mathrm{t}, J=7.6,2 \mathrm{H}, \mathrm{C}-\mathrm{CH}_{2}\right)$, 3.0 (t, $\left.J=7.7,2 \mathrm{H}, \mathrm{CH}_{2}-\mathrm{CO}\right), 5.30\left(\mathrm{~s}, 2 \mathrm{H},-\mathrm{N}-\mathrm{CH}_{2}-\mathrm{N}-\right)$, 7.00-7.83 (m, 5H, Ar-H); ); ${ }^{13} \mathrm{C} \mathrm{NMR}\left(\mathrm{CDCl}_{3}\right) \delta(\mathrm{ppm})$ : $14.5\left(2 \mathrm{XCH}_{3}\right), 27.5,35,65.5\left(3 \mathrm{XCH}_{2}\right), 128.1-155(\mathrm{Ar}-\mathrm{C})$, $175(\mathrm{C}=\mathrm{O}) ; \mathrm{MS}(\mathrm{m} / \mathrm{z}): 283\left(\mathrm{M}^{+} ; \mathrm{C}_{15} \mathrm{H}_{17} \mathrm{~N}_{5} \mathrm{O}\right)$.

6-naphthalen-2-yl-2-(1,2,4)-triazol-1-yl-methyl-4,5-

dihydro $(2 \mathrm{H})$-pyridazin-3-one (3h)

Yield: $40 \%$; mp $141-144^{\circ} \mathrm{C}$; IR $\left(\mathrm{KBr}, \mathrm{cm}^{-1}\right): 3015$ $(\mathrm{CH}), 1686(\mathrm{C}=\mathrm{O}) .{ }^{1} \mathrm{H} \mathrm{NMR}\left(\mathrm{CDCl}_{3}\right) \delta(\mathrm{ppm}): 2.68(\mathrm{t}, 2 \mathrm{H}$, $\left.\mathrm{C}-\mathrm{CH}_{2}\right), 2.98\left(\mathrm{t}, 2 \mathrm{H}, \mathrm{CH}_{2}-\mathrm{CO}\right), 5.26\left(\mathrm{~s}, 2 \mathrm{H},-\mathrm{N}-\mathrm{CH}_{2}-\mathrm{N}-\right)$, 7.3-8.3 (m, 9H, $\mathrm{Ar}-\mathrm{H}) ;{ }^{13} \mathrm{C} \mathrm{NMR}\left(\mathrm{CDCl}_{3}\right) \delta(\mathrm{ppm}): 27.2$, 34.2, 67.1 $\left(3 \mathrm{XCH}_{2}\right), 125.1-154.9(\mathrm{Ar}-\mathrm{C}), 176.1(\mathrm{C}=\mathrm{O})$; $\mathrm{MS}$ $(\mathrm{m} / \mathrm{z}): 305\left(\mathrm{M}^{+} ; \mathrm{C}_{17} \mathrm{H}_{15} \mathrm{~N}_{5} \mathrm{O}\right)$.

6-p-tolyl-2-(1,2,4)-triazol-1-yl-methyl-4,5- dihydro(2H) -pyridazin-3-one (3i)

Yield: 52\%; mp 138-140 ${ }^{\circ} \mathrm{C}$; IR (KBr, cm $\left.{ }^{-1}\right): 2995$ $(\mathrm{CH}), 1676(\mathrm{C}=\mathrm{O}) .{ }^{1} \mathrm{H}$ NMR $\left(\mathrm{CDCl}_{3}\right) \delta(\mathrm{ppm}): 2.31(\mathrm{~s}$, $\left.3 \mathrm{H}, \mathrm{CH}_{3}\right), 2.66$ (t, 2H, C- $\mathrm{CH}_{2}$ ), 3.0 (t, 2H, $\left.\mathrm{CH}_{2}-\mathrm{CO}\right), 5.3$ (s, 2H, -N- $\left.\mathrm{CH}_{2}-\mathrm{N}-\right)$, 6.87-7.83 (m, 6H, Ar-H); ${ }^{13} \mathrm{C} \mathrm{NMR}$ $\left(\mathrm{CDCl}_{3}\right) \delta(\mathrm{ppm}): 21.1\left(\mathrm{CH}_{3}\right), 27.2,34.9,66.1\left(3 \mathrm{XCH}_{2}\right)$, 128.5-155.5 (Ar-C), $174.8(\mathrm{C}=\mathrm{O}) ; \mathrm{MS}(\mathrm{m} / \mathrm{z}): 269\left(\mathrm{M}^{+}\right.$; $\left.\mathrm{C}_{14} \mathrm{H}_{15} \mathrm{~N}_{5} \mathrm{O}\right)$.

2(imidazol-1-yl-methyl)-6-p-tolyl-4,5- dihydro $(2 \mathrm{H})$ pyridazin-3-one (3j)

Yield: $45 \%$; mp $122-123{ }^{\circ} \mathrm{C}$; IR $\left(\mathrm{KBr}, \mathrm{cm}^{-1}\right): 3050$ $(\mathrm{CH}), 1700(\mathrm{C}=\mathrm{O}) .{ }^{1} \mathrm{H}$ NMR $\left(\mathrm{CDCl}_{3}\right) \delta(\mathrm{ppm}): 2.33(\mathrm{~s}$, $\left.3 \mathrm{H}, \mathrm{CH}_{3}\right), 2.66\left(\mathrm{t}, 2 \mathrm{H}, \mathrm{C}-\mathrm{CH}_{2}\right), 3.0\left(\mathrm{t}, 2 \mathrm{H}, \mathrm{CH}_{2}-\mathrm{CO}\right), 5.3$ (s, $\left.2 \mathrm{H},-\mathrm{N}-\mathrm{CH}_{2}-\mathrm{N}-\right), 6.88-7.83(\mathrm{~m}, 7 \mathrm{H}, \mathrm{Ar}-\mathrm{H}) ;{ }^{13} \mathrm{C} \mathrm{NMR}$ $\left(\mathrm{CDCl}_{3}\right) \delta(\mathrm{ppm}): 20.2\left(\mathrm{CH}_{3}\right), 27.7,33.7,65.1\left(3 \mathrm{XCH}_{2}\right)$, 122.5-151.9 (Ar-C), $173.5(\mathrm{C}=\mathrm{O})$; $\mathrm{MS}(\mathrm{m} / \mathrm{z}): 268\left(\mathrm{M}^{+}\right.$; $\left.\mathrm{C}_{15} \mathrm{H}_{16} \mathrm{~N}_{4} \mathrm{O}\right)$.

\section{Pharmacology}

The in vivo anticonvulsant activity was investigated by using Swiss albino mice (20-30 g) of either sex. The experimental animals were provided standard diet, free access to food and water and were housed in plastic cages 
maintained at room temperature $\left(25-30^{\circ} \mathrm{C}\right)$. The animals were divided into the groups having 5 animals in each. A prior approval for conducting the study was obtained from the Institutional Animal Ethics Committee (IAEC) of GRD (PG) IMT, Dehradun, India.

\section{Neurotoxicity screening}

Rotarod performance test was used to measure the synthesized molecules' interference with the motor coordination activity. For this purpose, mice were acclimated and trained to stay on the rotarod prior to the experiment. The rod having a diameter of $3.2 \mathrm{~cm}$ was operated at 10 rotations $\mathrm{min}^{-1}$. Normally a mouse could maintain equilibrium on the rotating rod by using its fore limbs and hind limbs for prolonged period of time. The title compounds 3a-j (50 and $100 \mathrm{mg} \cdot \mathrm{kg}^{-}{ }^{1}$ body mass) were injected intraperitoneally (i.p.) and their effect on impairment of motor activity was measured. Neurotoxicity of tested compounds was signaled by the inability of the animal to maintain equilibrium (balance) on the rod for at least one minute in each of the three attempts. Mice lose their balance due to the relaxation of skeletal muscle caused by the drug (Sethi et al., 2011).

\section{Anticonvulsant screening}

\section{Maximum electroshock seizure (MES) method}

The anticonvulsant screening of the test compounds (3a-j) was assessed as per the standard drug development protocol for anticonvulsant agents. Albino mice having previously received the test/standard drug (50 mg. $\mathrm{kg}^{-1}$ body mass) by i.p. route were stimulated through $50 \mathrm{~mA}$ current of corneal electrodes for $0.20 \mathrm{sec}$ at a pulse of $60 \mathrm{~Hz}$. Anticonvulsant activity was indicated by the abolition of the hind limb tonic extension spasm and was recorded (Kulkarni, 1999; Porter et al., 1984). The standard drugs; sodium valproate $\left(100 \mathrm{mg} . \mathrm{kg}^{-1}\right)$ and phenytoin sodium ( $25 \mathrm{mg} \cdot \mathrm{kg}^{-1}$ ) were used for comparing the anticonvulsant activity.

\section{Isoniazid (INH) induced seizures method}

To evaluate the anticonvulsant activity of the synthesized compounds by Isoniazid (INH) induced seizures test method, the compounds were administered in mice at a dose of $50 \mathrm{mg} \cdot \mathrm{kg}^{-1}$ i.p. After $30 \mathrm{~min}$ of the test drug administration, all mice received an i.p. injection of INH (250 mg. $\left.\mathrm{kg}^{-1}\right)$. The sequence of seizure latency, no. of seizures and $\%$ protection were studied. The reference drugs used in this model for comparison purpose were sodium valproate $\left(100 \mathrm{mg} \cdot \mathrm{kg}^{-1}\right)$ and phenytoin sodium (25 mg.kg-1) (Porter et al., 1984; Sethi et al., 2011).
Pentylenetetrazole (PTZ) induced seizures method

To assess the anticonvulsant activity of the synthesized compounds by Pentylenetetrazole (scPTZ) induced seizures test method, mice were injected with test drugs $50 \mathrm{mg} \cdot \mathrm{kg}^{-1}$ i.p. After $30 \mathrm{~min}$, all mice received subcutaneous (s.c.) injection of scPTZ at dose of $80 \mathrm{mg} \cdot \mathrm{kg}^{-1}$. The sequence of seizure latency, no. of seizures and \% protection were studied. The standard drugs used in this model were sodium valproate $\left(100 \mathrm{mg} \cdot \mathrm{kg}^{-1}\right)$ and phenytoin sodium $\left(25 \mathrm{mg} \cdot \mathrm{kg}^{-1}\right)$ (Porter et al., 1984; Sethi et al., 2011).

\section{Strychnine (STR) induced seizures method}

For the evaluation of anticonvulsant activity against strychnine (STR) induced convulsions, the same process was implemented as mentioned in PTZ induced convulsions. Instead, strychnine sulphate (STR-3 mg. $\mathrm{kg}^{-1}$ i.p.) was used for inducing convulsions (Porter et al., 1984; Sethi et al., 2011).

\section{Anti-TB activity by using Microplate Alamar Blue Dye} test

The anti-tubercular activity of synthetic compounds (3a-j) was tested against M. tuberculosis using a non toxic microplate Alamar Blue screening assay (MABA) technique. A thermally stable reagent is used in rapid and safer MABA technique which demonstrates good correlation and proportionality with BACTEC radiometric assay method. Briefly, $200 \mu \mathrm{L}$ of sterile deionized water was added to all outer perimeter wells of sterile 96 wells plate to reduce the evaporation of medium from the test wells during incubation. A measured $100 \mu \mathrm{L}$ of the Middle brook $7 \mathrm{H} 9$ broth was added to the 96 wells plate and was used directly to prepare the serial dilution of compounds. The final drug concentrations of $\mathbf{3} \mathbf{a}-\mathbf{j}$ were kept between 100 to $0.2 \mu \mathrm{g} / \mathrm{mL}$ for determination of MIC. The sealed and covered plates with parafilm were incubated at $37^{\circ} \mathrm{C}$ for five days. After five days, $25 \mu \mathrm{L}$ of freshly prepared 1:1 mixture of Almar Blue reagent and 10\% tween 80 were added to the plate and again incubated for $24 \mathrm{~h}$. Appearance of blue color in the well was inferred as absence of bacterial growth, whereas a pink color was scored as growth. The lowest concentration which prevented color change from blue to pink was considered as the MIC (Lourenco et al., 2007).

\section{Statistical analysis}

All the tests were performed in triplicate and results are expressed as mean \pm S.E.M. One-way analysis of variance (ANOVA) followed by Tukey's test was used to determine the statistical significance among groups. A $p$-value $(<0.05)$ was considered statistically significant. 


\section{RESULTS AND DISCUSSION}

The chemical structures of prepared pyridazinone compounds (3a-j) (Table I) were characterized on the basis of spectral data. The IR spectra revealed presence of $\mathrm{C}=\mathrm{O}$ and $\mathrm{CH}$ groups. The ${ }^{1} \mathrm{H}$ NMR showed singlet for methyl and two signals with appropriate multiplicities for ethyl groups attached to phenyl ring and two triplets for methylene protons at $4^{\text {th }}$ and $5^{\text {th }}\left(\mathrm{CH}_{2}-\mathrm{CH}_{2}\right)$ of pyridazinone ring, respectively. The singlet at $\delta 5.26-5.30$ is due to a methylene group flanked by two nitrogen atoms. In the

TABLE I - Structures of 6-aryl-2-(substituted amino-1yl)-2methyl-4,5- dihydro $(2 H)$-pyridazin-3-one derivatives (3a-j)

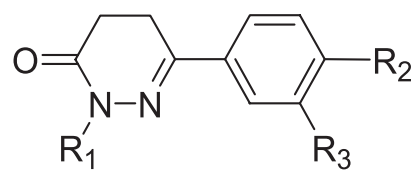

General Structure of 6-aryl-4,5-dihydro $(2 H)$ pyridazin-3one

3e 3

aromatic region, most of the compounds showed multiple peaks at $7-8 \delta$ value. The ${ }^{13} \mathrm{C}$ NMR and mass spectral data were also found in consistent with the chemical structure of synthesized compounds.

The final pyridazinone compounds (3a-j) were screened for the anticonvulsant activity by maximum electro shock (MES, $50 \mathrm{mg} \cdot \mathrm{kg}^{-1}$ ), isoniazid (INH, $250 \mathrm{mg} \mathrm{kg}^{-1}$ ), and pentylenetetrazole (scPTZ, $80 \mathrm{mg} \cdot \mathrm{kg}^{-1}$ ) induced seizures methods in mice. The results are shown in Table II-V and have been compared with the standard drugs, sodium valproate and phenytoin sodium. The title compounds (3a-j) were also tested for antitubercular activity by microplate alamar blue dye assay (MABA) method and the results are presented in Table VI.

None of the tested compounds showed neurotoxicity or impairment of motor coordination activity in mice by rotarod method at dose level of $100 \mathrm{mg} \cdot \mathrm{kg}^{-1}$. The MES, scPTZ and INH seizure models represent the three animal seizure models which are most reliable and thus routinely used in the screening of a new chemical entity as anticonvulsant agent.

In the present study, the MES seizure model involving extensor phase, which is similar to grand mal epilepsy, was used for preliminary screening of compounds $\mathbf{3 a - j}$. It can be seen that all the compounds $(\mathbf{3} \mathbf{a}-\mathbf{j})$ were able to prevent seizure spread in MES test method (Table II). Amongst all the compounds, six compounds $\mathbf{3 e}, \mathbf{3 c}, \mathbf{3 b}, \mathbf{3 i}, \mathbf{3 h}$ and $\mathbf{3 g}$ produced conspicuous anticonvulsant activity showing protection against MES-induced seizures at the dose of $50 \mathrm{mg} \cdot \mathrm{kg}^{-1}$. These six compounds are more potent than standard drug phenytoin sodium at the dose of $25 \mathrm{mg}$. $\mathrm{kg}^{-1}$, remaining compounds showed less protection than phenytoin sodium. It was interesting to note that majority of the screened compounds were observed to be more potent in comparison to the reference drug, sodium valproate at the dose of $100 \mathrm{mg} \cdot \mathrm{kg}^{-1}$. Thus, it could be suggested that the aryl-pyridazinone derivatives produce their action by inhibiting the voltage-gated ion channels (particularly sodium channels) analogous to a sodium channel inhibitor phenytoin which is quite active in MES model.

Most of the synthesized compounds (3a-j) exhibited protection against INH induced convulsions. The compounds after administration increased the onset of convulsion time from 36.2-47.6 min, reduced the number of convulsions (2.8 to 1.2) and increased the number of recovery of animals (80-100) in comparison to control group (26.16 min, 3.2 and 0 respectively). Compounds $\mathbf{3 f}$, $\mathbf{3 i}, \mathbf{3} \mathbf{j}, \mathbf{3} \mathbf{c}$ and $\mathbf{3 d}$ are noted to be more effective than the rest of the compounds. Compounds $\mathbf{3 f}$ showed maximum activity while compound $\mathbf{3 e}$ showed the lowest activity among all the synthetic compounds (3a-j) (Table III). 
TABLE II - Anticonvulsant activities of 6-aryl-2-(imidazol-1-yl / 1,2,4-triazol-1-yl)-2-methyl-4,5- dihydro (2H)-pyridazin-3-one against maximum electro shock (MES) induced method

\begin{tabular}{ccccccc}
\hline Compounds & $\begin{array}{c}\text { Flexion } \\
\text { (in sec) }\end{array}$ & $\begin{array}{c}\text { Extensor } \\
\text { (in sec) }\end{array}$ & $\begin{array}{c}\text { Clonus } \\
\text { (in sec) }\end{array}$ & $\begin{array}{c}\text { Stupor } \\
\text { (in sec) }\end{array}$ & $\begin{array}{c}\text { Recovery } \\
\text { (in sec) }\end{array}$ & Recovery \\
\hline Control & $6.8 \pm 0.10$ & $16.2 \pm 0.50$ & $16.5 \pm 0.29$ & $29.3 \pm 1.7$ & $225.4 \pm 9.61$ & 00 \\
3a & $6.4 \pm 0.50^{\gamma 3}$ & $4.4 \pm 0.50^{\mathrm{c}}$ & $15.2 \pm 1.06^{3}$ & $21.8 \pm 3.8^{\gamma}$ & $120.6 \pm 4.43^{1 \mathrm{a}}$ & 100 \\
$\mathbf{3 b}$ & $8.2 \pm 1.02^{\gamma 3 \mathrm{c}}$ & $2.4 \pm 0.50^{\gamma \mathrm{c}}$ & $20.5 \pm 1.6^{3 \mathrm{c}}$ & $20.3 \pm 2.6^{\gamma}$ & $117.3 \pm 7.52^{\mathrm{b}}$ & 80 \\
$\mathbf{3 c}$ & $12.0 \pm 0.70^{\gamma 3 \mathrm{c}}$ & $2.4 \pm 0.50^{\gamma \mathrm{c}}$ & $20 \pm 1.8^{3 \mathrm{c}}$ & $18 \pm 0.91^{\gamma}$ & $78 \pm 7.45^{\mathrm{c}}$ & 80 \\
$\mathbf{3 d}$ & $12.4 \pm 0.81^{\gamma 3 \mathrm{c}}$ & $4.0 \pm 0.83^{\mathrm{c}}$ & $9.0 \pm 1.0^{\mathrm{c}}$ & $49 \pm 7.0$ & $64.8 \pm 7.36^{\gamma 3 \mathrm{c}}$ & 80 \\
$\mathbf{3 e}$ & $4.6 \pm 1.32^{\mathrm{b}}$ & $2.0 \pm 0.63^{\gamma \mathrm{c}}$ & $8.5 \pm 0.50^{\mathrm{c}}$ & $15 \pm 3.0$ & $67.2 \pm 5.99^{\mathrm{c}}$ & 100 \\
$\mathbf{3 f}$ & $9.2 \pm 1.46^{\gamma 3 \mathrm{~b}}$ & $6.0 \pm 1.15^{3 \mathrm{c}}$ & $13.5 \pm 1.5^{3 \mathrm{~b}}$ & $18 \pm 2.00^{\gamma 3}$ & $39 \pm 13.06^{\mathrm{c}}$ & 100 \\
$\mathbf{3 g}$ & $2.2 \pm 0.37^{\mathrm{c}}$ & $3.0 \pm 1.0^{\gamma \mathrm{c}}$ & $11 \pm 1.0^{\beta 3 \mathrm{c}}$ & $21 \pm 3.0^{\gamma 3}$ & $40.8 \pm 7.54^{\mathrm{b}}$ & 100 \\
$\mathbf{3 h}$ & $6.8 \pm 0.86^{\gamma 3}$ & $2.8 \pm 0.37^{\gamma \mathrm{c}}$ & $9.0 \pm 1.0^{\mathrm{c}}$ & $14 \pm 2.0$ & $35.8 \pm 8.05^{\mathrm{c}}$ & 100 \\
$\mathbf{3 i}$ & $5.4 \pm 0.74^{\alpha 3}$ & $2.8 \pm 0.47^{\gamma \mathrm{c}}$ & $14 \pm 1.0^{\mathrm{a}}$ & $16 \pm 2.0$ & $41.8 \pm 9.14^{\mathrm{c}}$ & 80 \\
$\mathbf{3 j}$ & $2.8 \pm 0.58^{\mathrm{c}}$ & $4.5 \pm 0.50^{\mathrm{c}}$ & $7.0 \pm 1.0^{\mathrm{c}}$ & $13.5 \pm 1.5$ & $63.3 \pm 4.9^{\mathrm{c}}$ & 80 \\
Phenytoin Sod & $3.1 \pm 0.20$ & $3.3 \pm 0.56$ & $6.7 \pm 0.51$ & $14.2 \pm 0.82$ & $25.0 \pm 2.1$ & 100 \\
SodValproate & $3.6 \pm 0.82$ & $5.3 \pm 0.68$ & $7.6 \pm 1.24$ & $18.8 \pm 2.43$ & $26 \pm 3.31$ & 80 \\
\hline
\end{tabular}

Standard drug: Phenytoin sodium (25mg.kg $\left.{ }^{-1}\right)$ and sodium valproate $\left(100 \mathrm{mg} \cdot \mathrm{kg}^{-1}\right)$, Tested drugs: Synthesized compounds 3a-g $\left(50 \mathrm{mg} \cdot \mathrm{kg}^{-1}\right)$, Control group: $0.5 \%$ Carboxyl methyl cellulose $(\mathrm{CMC})$ in distilled water; $\mathrm{n}=5$ (No. of animals in each group); *Value represents mean \pm S.E.M. ${ }^{\mathrm{a}} P<0.05,{ }^{\mathrm{b}} P<0.01$ and ${ }^{\mathrm{c}} P<0.001$ when compared to control group; ${ }^{\alpha} \mathrm{P}<0.05,{ }^{\beta} \mathrm{P}<0.01$ and ${ }^{\gamma} \mathrm{P}<0.001$ when compared to standard drug sodium valproate; ${ }^{1} P<0.05,{ }^{2} P<0.01$ and ${ }^{3} P<0.001$ when compared to standard drug phenytoin sodium

TABLE III - Anticonvulsant activities of 6-aryl-2-(imidazol-1-yl /1,2,4-triazol-1-yl)-2-methyl-4,5- dihydro (2H)-pyridazin-3-one against isoniazid (INH) induced method

\begin{tabular}{cccc}
\hline Compounds & $\begin{array}{c}\text { Onset of } \\
\text { convulsion }\end{array}$ & $\begin{array}{c}\text { No. of } \\
\text { convulsion }\end{array}$ & Recovery \\
\hline $\mathbf{3 a}$ & $36.2 \pm 9.27^{\gamma \mathrm{a}}$ & $1.7 \pm 0.25$ & 100 \\
$\mathbf{3 b}$ & $46 \pm 4.51^{\gamma \mathrm{c}}$ & $2.6 \pm 0.24$ & 80 \\
$\mathbf{3 c}$ & $45.6 \pm 4.87^{\gamma \mathrm{c}}$ & $2.8 \pm 0.20$ & 80 \\
$\mathbf{3 d}$ & $46.6 \pm 3.21^{\text {c }}$ & $2.2 \pm 0.37$ & 100 \\
$\mathbf{3 e}$ & $35.2 \pm 8.98^{\gamma}$ & $2.75 \pm 0.25$ & 100 \\
$\mathbf{3 f}$ & $47.6 \pm 1.6^{\gamma \mathrm{c}}$ & $2.2 \pm 0.37$ & 100 \\
$\mathbf{3 g}$ & $41.8 \pm 2.03^{\gamma \mathrm{c}}$ & $2.0 \pm 0.44$ & 100 \\
$\mathbf{3 h}$ & $48 \pm 1.7^{\gamma \mathrm{c}}$ & $2.8 \pm 0.20$ & 80 \\
$\mathbf{3 i}$ & $46.6 \pm 4.04^{\gamma \mathrm{c}}$ & $2.4 \pm 0.40$ & 100 \\
$\mathbf{3 j}$ & $47.2 \pm 4.58^{\gamma \mathrm{c}}$ & $2.2 \pm 0.20$ & 100 \\
Sodium valproate & $130.2 \pm 2.15$ & $1.2 \pm 0.20$ & 100 \\
Phenytoin sodium & 0.00 & 0.00 & 100 \\
Control & $26.16 \pm 0.94$ & $3.2 \pm 0.20$ & 00 \\
\hline
\end{tabular}

Standard drug: Phenytoin sodium (25 mg. $\left.\mathrm{kg}^{-1}\right)$ and sodium valproate (100 mg. $\left.\mathrm{kg}^{-1}\right)$, Tested drugs: Synthesized compounds 3a-j (50 mg. $\mathrm{kg}^{-1}$ ), Control group: INH-250 mg. $\mathrm{kg}^{-1}+0.5 \%$ carboxyl methyl cellulose (CMC) in distilled water; $n=5$ (No. of animals in each group); *Value represents mean \pm S.E.M. ${ }^{\mathrm{a}} P<0.05,{ }^{\mathrm{b}} P<0.01$ and ${ }^{\mathrm{c}} P<0.001$ when compared to control group; ${ }^{\alpha} P<0.05,{ }^{\beta} \mathrm{P}<0.01$ and ${ }^{\gamma} P<0.001$ when compared to standard drug sodium valproate
In PTZ induced convulsion model, compounds 3a, $\mathbf{3 d}, \mathbf{3 j}, \mathbf{3 g}$ and $\mathbf{3 h}$ showed good anticonvulsant activity. The control group showed an onset of convulsion, number of convulsions and recovery as $7.6 \mathrm{~min}, 1.8$ and 0 respectively. In contrast, groups treated with the synthetic compounds had the onset of convulsions ranging from 13.4 to $16.6 \mathrm{~min}$ and indicate protection. It can also be seen from the results that the synthesized compounds decreased the number of convulsions (1-1.6) and lead to the better recovery of animals (60-100\%). Compound 3a (16.6 min) showed the highest activity and compound $\mathbf{3 b}$ (13.4 $\mathrm{min}$ ) showed the lowest activity amongst all the screened compounds $(\mathbf{3 a}-\mathbf{j})$ in terms of onset of convulsions (Table IV). Similarly, compound 3h, 3i (1.0), 3a, 3b, 3c, $\mathbf{3 f}, \mathbf{3 j}$ (1.2) produced lower number of convulsions in comparison to standard drug sodium valproate (1.3). The recovery rate of $\mathbf{3 h}, \mathbf{3 i}$ and $\mathbf{3} \mathbf{j}$ was $100 \%$ while it was found to be $60 \%$ for $3 \mathrm{~g}$. However, the title compounds were having a weak anticonvulsant spectrum in comparison to Phenytoin sodium.

In STR-induced convulsions ( $\left.3 \mathrm{mg} \cdot \mathrm{kg}^{-1}\right)$, compounds (3a-j) at $50 \mathrm{mg} \cdot \mathrm{kg}^{-1}$ dose level did not show any significant anticonvulsant activity (Table V). Compounds 3a-j did not show any significant decrease in the number of convulsions (2.6-3.0) and onset of convulsions (1.92-2.96) induced by the administration of STR in comparison to control (3.17 and 1.5 respectively). It is well know that seizures produced by PTZ and INH are due to the inhibition of 
TABLE IV - Anticonvulsant activities of 6-aryl-2-(imidazol-1-yl / 1,2,4-triazol-1-yl)-2-methyl-4,5- dihydro $(2 H)$-pyridazin-3-one against pentyleneteterzole (PTZ) induced method

\begin{tabular}{cccc}
\hline Compounds & $\begin{array}{c}\text { Onset of } \\
\text { convulsion } \\
\text { (min) }\end{array}$ & $\begin{array}{c}\text { No. of } \\
\text { convulsion }\end{array}$ & Recovery \\
\hline Control & $7.6 \pm .74$ & $1.8 \pm 0.44$ & 00 \\
3a & $16.6 \pm 0.92^{\gamma c}$ & $1.2 \pm 0.44$ & 80 \\
3b & $13.4 \pm 1.07^{\gamma c}$ & $1.2 \pm 0.44$ & 80 \\
$\mathbf{3 c}$ & $14.4 \pm 0.81^{\gamma c}$ & $1.2 \pm 0.44$ & 60 \\
$\mathbf{3 d}$ & $16.2 \pm 1.06^{\gamma \mathrm{c}}$ & $1.4 \pm 0.54$ & 80 \\
$\mathbf{3 e}$ & $14.6 \pm 0.74^{\gamma \mathrm{c}}$ & $1.6 \pm 0.54$ & 80 \\
$\mathbf{3 f}$ & $14 \pm 0.70^{\gamma \mathrm{c}}$ & $1.2 \pm 0.44$ & 80 \\
$\mathbf{3 g}$ & $15 \pm 1.04^{\gamma \mathrm{c}}$ & $1.4 \pm 0.54$ & 60 \\
$\mathbf{3 h}$ & $14.8 \pm 0.44^{\gamma \mathrm{c}}$ & $1 \pm 0.00$ & 100 \\
$\mathbf{3 i}$ & $14.2 \pm 0.86^{\gamma \mathrm{c}}$ & $1 \pm 0.00$ & 100 \\
$\mathbf{3 j}$ & $15.2 \pm 0.66^{\gamma \mathrm{c}}$ & $1.2 \pm 0.44$ & 100 \\
Sodium valproate & $68.2 \pm 1.54$ & $1.3 \pm 1.4$ & 100 \\
Phenytoin Sodium & 0.00 & 0.00 & 100 \\
\hline Standard
\end{tabular}

Standard drug: Phenytoin sodium (25 mg. $\left.\mathrm{kg}^{-1}\right)$ and sodium valproate (100 mg.kg-1), Tested drugs: Synthesized compounds 3a-j (50 mg. $\left.\mathrm{kg}^{-1}\right)$, Control group: PTZ-80 mg.kg-1 $+0.5 \%$ carboxyl methyl cellulose (CMC) in distilled water; $n=5$ (No. of animals in each group); *Value represents mean \pm S.E.M. ${ }^{\mathrm{a}} P<0.05,{ }^{\mathrm{b}} P<0.01$ and ${ }^{\mathrm{c}} P<0.001$ when compared to control group; ${ }^{\alpha} P<0.05,{ }^{\beta} P<0.01$ and ${ }^{\gamma} P<0.001$ when compared to standard drug sodium valproate.

$\gamma$-aminobutyric acid (GABA) neurotransmission in the brain (Kalso, 2005; Dalkara, Karakurt, 2012; Zulini, Fantini, Rivara, 2012). GABA is the primary inhibitory neurotransmitter in the central nervous system, and its deficiency or inhibition is linked to genetic and acquired epilepsy (Gajcy, Lochynski, Librowski, 2010; Dalkara, Karakurt, 2012). While increased GABAergic neurotransmission inhibit or decrease seizure propensity. It was observed that compounds $\mathbf{3} \mathbf{a}-\mathbf{j}$ thoroughly inhibited the clonic seizures, tonic seizures and lethality induced by sc-PTZ and INH, while the reference drugs also inhibit the clonic seizures induced by sc-PTZ and INH (Table III and Table IV). Those compounds which exhibited high anticonvulsant activity in the MES and sc-PTZ models are most likely to possess a good anticonvulsant profile and can be explored further for drug development. The STR (glycine antagonist) test is known to be sensitive to antagonize glycine (Dutertre, Becker, Heinrich Betz, 2012), which suggested that compounds 3a-j may not inhibit the glycine concentration in the brain.

\section{Possible mechanisms of anticonvulsant compounds}

The commonly used in vivo models for preclinical discovery and development of new drug molecule for the treatment of epilepsy include the MES, scPTZ, INH and STR models. Amongst them, the MES and scPTZ seizure models are the most preferred animal models for the screening of AEDs (Kupferberg, 2001). The MES test is quite sensitive to sodium $\left(\mathrm{Na}^{+}\right)$channel inhibitors (e.g. Phenytoin), which suggested that aryl-pyridazinone compounds are producing their actions by inhibiting voltage-gated ion channels (particularly $\mathrm{Na}^{+}$channels). The INH (GABA synthesis inhibitor) and the PTZ (GABA antagonist) test are known to be sensitive to modulate $\gamma$-amino butyric acid (GABA), which suggested that synthesized compounds may increase the concentration of inhibitory neurotransmitter, GABA in the brain. The STR (Glycine antagonist) test is known to be sensitive to antagonize glycine, which suggests that compounds may not inhibit the glycine concentration in the brain. Thus, it could be proposed that the title compounds could be acting as voltage-gated ion channel (mainly $\mathrm{Na}^{+}$-channel) inhibitors and GABA modulators in the brain.

TABLE V - Anticonvulsant activities of 6-aryl-2-(imidazol-1-yl / 1,2,4-triazol-1-yl)-2-methyl-4,5- dihydro(2H)-pyridazin-3-one (3a-j) against STR-induced convulsion method

\begin{tabular}{cccc}
\hline Compounds & $\begin{array}{c}\text { Onset of } \\
\text { convulsion } \\
\text { (min) }\end{array}$ & $\begin{array}{c}\text { No. of } \\
\text { convulsion }\end{array}$ & Recovery (\%) \\
\hline $3 \mathrm{a}$ & $2.54 \pm 0.34^{3}$ & $2.72 \pm 0.23^{3}$ & 00 \\
$3 \mathrm{~b}$ & $2.60 \pm 0.38^{3}$ & $2.80 \pm 0.15^{3}$ & 00 \\
$3 \mathrm{c}$ & $2.82 \pm 0.40^{\mathrm{a3}}$ & $2.76 \pm 0.15^{3}$ & 00 \\
$3 \mathrm{~d}$ & $1.92 \pm 0.29^{3}$ & $3.0 \pm 0.16^{3}$ & 00 \\
3e & $2.18 \pm 0.076^{\mathrm{a3}}$ & $2.60 \pm 0.20^{3}$ & 00 \\
3f & $2.88 \pm 1.09^{\mathrm{a} 2}$ & $2.74 \pm 2.18^{3}$ & 00 \\
$3 \mathrm{~g}$ & $2.96 \pm 0.320^{\mathrm{a3}}$ & $2.74 \pm 2.18^{3}$ & 00 \\
$3 \mathrm{~h}$ & $3.12 \pm 0.39^{\mathrm{a3}}$ & $2.74 \pm 2.18^{3}$ & 00 \\
$3 \mathrm{i}$ & $2.76 \pm 0.40^{\mathrm{a3}}$ & $2.60 \pm 0.20^{3}$ & 00 \\
$3 \mathrm{j}$ & $2.11 \pm 0.076^{\mathrm{a} 3}$ & $2.80 \pm 0.15^{3}$ & 00 \\
Control & $1.50 \pm 1.09$ & $3.17 \pm 2.18$ & 00 \\
Sod VPA & -- & -- & 100 \\
PHT sod & $8.45 \pm 1.86$ & $1.06 \pm 0.78$ & 40 \\
\hline
\end{tabular}

Standard drug: PHT sod (25 mg.kg-1 $)$ and sod VPA (50 mg.kg $\left.{ }^{-1}\right)$; Tested drugs: Synthesized compounds 3a-j $\left(50 \mathrm{mg} \cdot \mathrm{kg}^{-1}\right)$, Control group: STR $3 \mathrm{mg} \cdot \mathrm{kg}^{-1}+0.5 \% \mathrm{CMC}$ in distilled water; $\mathrm{n}=5$ (No. of animals/group). ${ }^{\mathrm{a}} P<0.05,{ }^{\mathrm{b}} P<0.01$ and ${ }^{\mathrm{c}} P<0.001$ when compared to control group. ${ }^{1} P<0.05,{ }^{2} P<0.01$ and ${ }^{3} P<0.001$ when compared to PHT sod. 
The minimum inhibitory concentration (MIC) values of the screened compounds against mycobacterium were determined by a standard procedure. The MIC values were observed in the range of 1.6-6.25 $\mu \mathrm{g} / \mathrm{mL}$. Compounds 3a, $\mathbf{3 b}, \mathbf{3 f}$ and $\mathbf{3 g}$ emerged as highly potent molecules in this series with MIC value of $1.6 \mu \mathrm{g} / \mathrm{mL}$, moderate activity were shown by compounds $\mathbf{3 c}, \mathbf{3 d}, \mathbf{3 e}$ and $\mathbf{3 i}$ with MIC value $3.125 \mu \mathrm{g} / \mathrm{mL}$ and lower activity was displayed by compounds $3 \mathbf{h}$ and $\mathbf{3 j}$ with MIC values $6.25 \mu \mathrm{g} / \mathrm{mL}$ against M. tuberculosis $\mathrm{H} 37 \mathrm{Rv}$ comparable with those of standard PZA (3.125 $\mu \mathrm{g} / \mathrm{mL})$ and STM $(6.25 \mu \mathrm{g} / \mathrm{mL})$ (Table VI). The results indicate that the pyridazine with 1,2,4-triazol1-yl-methyl and 4-ethylphenyl (3a), 6-biphenyl-4-yl and 2-imidazol-1-yl-methyl (3b), 3,4-dimethyl-phenyl and imidazol-1-ylmethyl (3f) and 1,2,4-triazol-1-yl-methyl and 3,4-dimethylphenyl (3g) group showed comparable activity with the reference drugs.

TABLE VI - MIC values for antitubercular activity of 6-aryl2-(imidazol-1-yl / 1,2,4-triazol-1-yl)-2-methyl-4,5- dihydro (2H)-pyridazin-3-one

\begin{tabular}{cccc}
\hline Compounds & $\begin{array}{c}\text { MIC } \\
(\boldsymbol{\mu g} / \mathbf{m L})\end{array}$ & Compounds & $\begin{array}{c}\text { MIC } \\
(\boldsymbol{\mu g} / \mathbf{m L})\end{array}$ \\
\hline 3a & 1.6 & $\mathbf{3 f}$ & 1.6 \\
$\mathbf{3 b}$ & 1.6 & $\mathbf{3 g}$ & 1.6 \\
$\mathbf{3 c}$ & 3.125 & $\mathbf{3 h}$ & 6.25 \\
$\mathbf{3 d}$ & 3.125 & $\mathbf{3 i}$ & 3.125 \\
$\mathbf{3 e}$ & 3.125 & $\mathbf{3 j}$ & 6.25 \\
Pyrazinamide (PZA) & 3.125 & & \\
Streptomycin (STM) & 6.25 & & \\
\hline
\end{tabular}

\section{CONCLUSIONS}

A new library of aryl-pyridazinone derivatives were synthesized by taking a lead from the existing drugs. The newly synthesized 6-aryl-2-(imidazol-1yl / 1,2,4-triazol-1-yl)-2-methyl-4,5- dihydro $(2 H)$ pyridazin-3-one (3a-j) exhibited potent anticonvulsant and antitubercular activities in the MES, PTZ, INH induced seizure and MABA test, respectively. Notably a compound $\mathbf{3 g}$ showed better anticonvulsant activity and higher safety in MES model than marketed drugs sodium valproate and phenytoin sodium. All of these suggested that compounds $\mathbf{3 a - j}$ are likely to have several mechanisms of action including inhibition of voltagegated ion channels and GABAergic activity. All tested compounds showed moderate to good activity against strains of M. tuberculosis. The results demonstrate that both imidazol-yl-methyl and triazol-yl-methyl group at $2 \mathrm{~N}$-position of pyridazinone ring is a suitable for anticonvulsant as well as anti-tubercular activity.

\section{CONFLICT OF INTEREST STATEMENT}

The authors declare that there are no conflicts of interest.

\section{ACKNOWLEDGEMENTS}

The authors are thankful to GRD (PG) Institute of Management \& Technology, Dehradun, India, Sophisticated Analytical Instrument Facilities, Central Drug Research Institute, Lucknow, Uttar Pradesh, India for providing financial as well as technical support and facilities to carry out this work. Authors are also grateful to the Department of Microbiology, Maratha Mandal NGH Institute of Dental Sciences and Research Centre, Belgaum, India for screening antitubercular activity.

\section{REFERENCES}

Ahsan W, Safhi MM, Siddiqui N, Javed S, Alam MS, Azad B, et al. An Insight into the new anticonvulsant agents. Curr Topics Med Chem. 2012;12(9):1072-1092.

Akahane A, Katayama H, Mitsunaga T. Discovery of 6-oxo-3- (2-phenylpyrazolo[1,5-a]pyridin-3-yl)-1(6H)pyridazinebutanoic acid (FK 838): A novel non-xanthine adenosine A1 receptor antagonist with potent diuretic activity. J Med Chem. 1999;42(5):779-783.

Amin EN, Abdel-Alim AA, Abdel-Moty SG, El-Shorbagi AN, Abdel-Rahman MS. Synthesis of new 4,5-3(2H) pyridazinone derivatives and their cardiotonic, hypotensive, and platelet aggregation inhibition activities. Arch Pharm Res. 2010;33(1):25-46.

Asif M. A review of antimycobacterial drugs in development. Mini Rev Med Chem. 2012a;129(13):1404-1418.

Asif M. Some recent approaches of biologically active substituted pyridazine and phthalazine drugs. Curr Med Chem. 2012b;19(18):2984-2191.

Asif M, Singh A, Bilkanti L. In-vivo anticonvulsant and in-vitro antitubercular activity of methyl indole derivative of some 6-aryl-4, 5-Dihydropyridazin-3(2H)-ones and their expected anticonvulsant mechanisms. Iranian J Pharm Sci. 2013;9(1):6780 . 
Asif M, Singh A. Synthesis and anti-tubercular activity of 6-(4-Chloro/Methyl-phenyl)-4-arylidene-4,5-dihydropyridazin3(2H)-one derivatives against Mycobacterium tuberculosis. Lett Drug Design Discov. 2015;12(6):500-504.

Bialer M. New antiepileptic drugs that are second generation to existing antiepileptic drugs. Expert Opin Investig Drugs. 2006;15(6):637-647.

Dalkara S, Karakurt A. Recent progress in anticonvulsant drug research: strategies for anticonvulsant drug development and applications of antiepileptic drugs for non-epileptic central nervous system disorders. Curr Topics Med Chem. 2012;12(9):1033-1071.

Demirayak S, Karaburun AC, Beis R. Some pyrrole substituted aryl pyridazinone and phthalazinone derivatives and their antihypertensive activities. Eur J Med Chem. 2004; 39(12):10891095.

Deng XQ, Song MX, Wei CX, Li FN, Quan ZS. Synthesis and anticonvulsant activity of 7-alkoxy-triazolo-(3,4-b)benzo(d) thiazoles. Med Chem. 2010;6(5):313-320.

Dutertre S, Becker CM, Heinrich Betz H. Inhibitory glycine receptors: An update. J Biol Chem. 2012;287(48):40216-40223.

Edafiogho IO, Phillips OA, Udo EE, Samuel S, Rethish B. Synthesis, antibacterial and anticonvulsant evaluations of some cyclic enaminones. Eur J Med Chem. 2009;44(3):967-975.

Gajcy K, Lochynski S, Librowski TA. Role of GABA analogues in the treatment of neurological diseases. Curr Med Chem. 2010;17(22):2338-2347.

Gerlach AC, Krajewski JL. Antiepileptic drug discovery and development: What have we learned and where are we going? Pharmaceuticals. 2010;3(9):2884-2899.

Ghogare JG, Bhandari SV, Bothara KG, Madgulkar AR, Parashar GA, Sonawane BG, et al. Design, synthesis and pharmacological screening of potential anticonvulsant agents using hybrid approach. Eur J Med Chem. 2010;45(3):857-863.

Gunia A, Waszkielewicz AM, Cegla M, Marona H. Preliminary evaluation of anticonvulsant activity of some aminoalkanol and amino acid cinnamic acid derivatives. Lett Drug Design Discov. 2010;9(1):37-43.
Hallot A, Brodin R, Merlier J, Brochard J, Chambon JP, Biziere K. Synthesis and activity of 6-aryl-3-(hydroxypolymethyleneamino) pyridazines in animal models of epilepsy. J Med Chem. 1986;29(3):369-375.

Hu C, Sun ZG, Wei CX, Quan ZS. Synthesis and Anticonvulsant activity of some cinnamylpiperazine derivatives. Lett Drug Design Discov. 2010;7(9):661-664.

Islam M, Siddiqui AA, Rajesh R. Synthesis, antitubercular, antifungal and antibacterial activities of 6 -substituted phenyl-2-(3'-substituted phenyl pyridazin-6'-yl)-2,3,4,5tetrahydropyridazin-3-one. Acta Pol Pharm. 2008;65(3):353362.

Kalso E. Sodium channel blockers in neuropathic pain. Curr Pharm Design. 2005;11(23):3005-3011.

Khokra SL, Seth S, Garg SS. Kaushik P, Ahmad A, Khan SA et al. Synthesis, computer aided screening and pharmacological evaluation of 2/3-substituted-6(4-methylphenyl)-4,5dihydropyridazin $3(2 \mathrm{~h})$-ones, and pyridazine substituted triazine. Indonesian J Pharm. 2015;26(4):200-209.

Kupferberg H. Animal models used in the screening of antiepileptic drugs. Epilepsia, 2001;42(Suppl 4):7-12.

Kulkarni SK. Handbook of experimental pharmacology. $3^{\text {rd }}$ edition. New Delhi: Vallabh publication; 1999. p.131-133.

Lourenco MCS, deSouza MVN, Pinheiro AC, Ferreira ML, Goncalves RB, Nogneira TCM, et al. Evaluation of antitubercular activity of nicotinic and isoniazid analogues. ARKIVOC. 2007;XV:181-191.

Sandhu GK. Tuberculosis: Current situation, challenges and overview of its control programs in India. J Glob Infect Dis. 2011;3(2):143-150.

Sethi KK, Verma SM, Prasanthi N, Annapurna MM. Synthesis, neurotoxicity and anticonvulsant study of some benzothiazole analogs. Lett Drug Design Discov. 2011;8(8):774-777.

Siddiqui AA, Abdullah MM, Arora M, Islam M, Ahmad SR. Synthesis of novel pyridazinones possessing anticonvulsant activity. Indian Drugs. 2006;43(10):790-794.

Siddiqui AA, Mishra R, Shaharyar M. Synthesis, characterization and antihypertensive activity of pyridazinone derivatives. Euro J Med Chem. 2010;45(6):2283-2290. 
Porter RJ, Cereghino JJ, Gladding GD, Hessie BJ, Kupferberg HJ, Scoville B, et al. Anti-epileptic drug development program. Cleve Clin Q. 1984;51:293-305.

Xu P, Wang SY, Chen Y, Liu WQ, Tao C. Studies on synthesis, anticonvulsant activity and the structure activity relationships of 6-(substituted phenyl)-3-(2H) pyridazinones. Yao Xue Xue Bao. 1991;26(9):656-660.
Zuliani V, Fantini M, Rivara M. Sodium channel blockers as therapeutic target for treating epilepsy: recent updates. Curr Topics Med Chem. 2012;12(9):962-970.

Received for publication on $16^{\text {th }}$ October 2016 Accepted for publication on $09^{\text {th }}$ January 2018 